\title{
Christoph Scherrer
}

\section{New Economy: Wachstumsschub durch Produktivitätsrevolution?}

Der Begriff New Economy ist ähnlich schillernd wie der Begriff der Moderne. Zum ersten Mal ist er mir in einem Aufsatz von Kim Moody begegnet (1994/1997), wo er für Deregulierung, schlanke Produktion und vor allem für eine ethnisch und nach Geschlecht neu zusammengesetzte Arbeiterschaft stand. Allgemeine Bekanntheit hat dieser Begriff durch eine Rede des Vorsitzenden des US-amerikanischen Zentralbankrates, Alan Greenspan, vor dem US-amerikanischen Kongress im Juli 1996 erhalten (Greenspan 1996). In dieser Rede begründete Greenspan den Verzicht auf Zinserhöhungen trotz sinkender Arbeitslosigkeit mit dem Hinweis auf hohe Produktivitätszuwächse: diese würde Preissteigerungen, die normalerweise mit einem hohen Beschäftigungsgrad einher gehen, verhindern. Mithin gälte die in den 80er Jahren weitverbreitete Annahme nicht mehr, dass die Preisstabilität bei einer Arbeitslosenquote von unter sechs Prozent gefährdet sei (NAIRU = non-accelerating inflation rate of unemployment). Preisstabilität könnte auch bei einer deutlich geringeren Arbeitslosigkeit gewahrt bleiben. Diese Situation sei neu, deshalb sei es berechtigt von einer New Economy zu sprechen. Als Hauptursache für das Produktivitätswachstum identifizierte Greenspan neben der verschärften internationalen Konkurrenz vor allem die Technologiesprünge in der Informationsbearbeitung.

„Our nation has been experiencing a higher growth rate of productivity - output per hour worked - in recent years. The dramatic improvement in computing power and communication and information technology appear to have been a major force behind this beneficial trend" (Greenspan 1998)

Im Sprachgebrauch wird der Begriff New Economy deshalb häufig auch für jene Wirtschaftszweige verwendet, die die auf das Internet bezogenen Informationstechnologien entwickeln, herstellen und anwenden (die sogenannte Internetökonomie; siehe Zerdick et al. 1999). Für die folgende Diskussion werde ich mich an Greenspans makroökonomischer Verwendung des Begriffes orientieren. Kennzeichnend für die New Economy in diesem Sinne ist, dass sie aufgrund hoher Produktivitätszuwächse ein dauerhaft höheres inflati- 
onsneutrales Wirtschaftswachstum zulässt (vgl. auch Bassanini et al. 2000). Inwieweit diese These zutrifft, ist Thema meines Beitrages. ${ }^{1}$

Für Greenspans These von der Existenz einer New Economy will ich zunächst die üblicherweise angeführten Belege vorstellen. So dann werde ich die Skeptiker kurz zu Wort kommen lassen, die die angeblichen Produktivitätszuwächse relativieren. Es folgt eine theoretische Begründung für Greenspans These, deren Ursprung in unverdächtiger Ferne zu den an dieser These interessierten Gruppen, wie der Clinton-Regierung, der Federal Reserve Board und den Wall-Street-Firmen liegt, nämlich in der französischen Regulationstheorie. ${ }^{2}$ Abschließend werde ich die empirische Basis für eine solche Deutung problematisieren und auf einige Trends verweisen, die auf die Vergänglichkeit und Singularität des derzeitig langanhaltenden Wirtschaftsaufschwunges in den USA hindeuten.

\section{Die politische Vorgeschichte der New Economy}

In Deutschland wurde der Begriff New Economy im Gefolge der Diskussionen um das so genannte Jobwunder in den USA zeitgleich mit dem Begriff Shareholder-Kapitalismus in den politischen Wortschatz aufgenommen. Entsprechend galt er als Kampfbegriff eines triumphierenden Kapitalismus (siehe die Kritik von Altvater und Mahnkopf 2000). Auch in den USA schwingt bei der Verwendung des Ausdrucks New Economy ein solcher Triumphalismus mit, doch zunächst diente der Begriff im Wahljahr 1996 zur Rechtfertigung eines wachstumsfördernden zinspolitischen Kurses der Zentralbank. Nachdem die Zentralbank von Beginn des Jahres 1994 bis zum Frühjahr 1995 die Zinsen von drei auf sechs Prozent heraufgesetzt hatte, befürchtete Präsident Clinton ein ähnliches Schicksal wie sein Vorgänger zu erleiden, nämlich Opfer eines nicht zuletzt zinspolitisch verlangsamten Wirtschaftswachstums zu werden. Bereits im Laufe des Jahres 1995 sprachen sich einige der Demokratischen Partei verbundene Ökonomen und Bankiers (insbesondere der New Yorker Investmentbanker Felix Rohatyn 1995), aber auch einige Industrielle für eine zinspolitische Wende aus (Woodward 2000: 150). Deren Argument, dass aufgrund des Einsatzes neuer Kommunikationstechnologien und der Erfolge des „schlanken Managements“ ein höheres inflationsfreies Wachstum möglich wäre, wurde von Präsident Clinton begeistert aufgenommen. Doch die zinspolitische Kompetenz lag beim Zentralbankausschuss für Offenmarktgeschäfte (Federal Open Market Committee), der vom Zentralbankvorsitzen-

1 Ich werde mich deshalb mit der Beschäftigungsseite des langen US-Aufschwunges nur am Rande auseinandersetzen. Eine umfassende, interdisziplinäre Analyse des sogenannten Jobwunders findet sich bei Lang et al. (1999).

$2 \mathrm{Zu}$ den Klassikern dieser französischen ökonomielastigen Gesellschaftstheorie gehören die Werke von Michel Aglietta (1979, 2000), Robert Boyer (1990) und Alain Lipietz (1985). Für eine Einführung aus ökonomietheoretischer Sicht siehe Hübner (1989). 
den Alan Greenspan dominiert wurde. Dieser marktwirtschaftlich orientierte Republikaner übernahm erstaunlicherweise das New-Economy-Argument. Ab Mitte 1995 senkte der Zentralbankausschuss in kurzer Folge die Zinsen auf 5 1/4\% und beließ sie während des gesamten Wahljahres 1996 auf diesem Niveau. Damit trug Greenspan nicht nur zum Wahlsieg von Präsident Clinton bei, sondern er verhalf auch dem ärmeren Teil der amerikanischen Gesellschaft zu Arbeit und Einkommen. Denn angesichts einer republikanischen Mehrheit in beiden Häusern des Kongresses bestand für Präsident Clinton keine Möglichkeit, durch Umverteilungspolitik Armut zu bekämpfen. Allein durch ein beschleunigtes Wirtschaftswachstum bestand Aussicht, denjenigen unter der traditionellen Demokratischen Anhängerschaft zu helfen, deren Stellung am Arbeitsmarkt prekär ist. So ist es nicht erstaunlich, dass progressive Ökonomen wie Barry Bluestone (1999) und Robert Eisner (1998) das Argument von der New Economy euphorisch übernehmen, während Anhänger der NAIRU wie Robert Gordon dem Argument eher skeptisch gegenüberstehen (siehe unten).

\section{Die These von der New Economy}

Im Kern beinhaltet die These von der New Economy die Behauptung, dass sich seit einigen Jahren ein höherer, nicht-inflationärer Wachstumstrend aufgrund eines insbesondere den Informationstechnologien geschuldeten höheren Produktivitätswachstums abzeichnet. Noch einmal in den Worten des Zentralbankchefs Alan Greenspan lautet die These folgendermaßen:

„It's certainly become increasingly difficult to deny that something profoundly different from typical post war business cycle has emerged in recent years. ... Most remarkably, inflation has remained subdued in the face of labor markets tighter than we experienced in at least a generation... While there are various competing explanations ..., the most compelling appears to be the extraordinary surge in technological innovation ... In the early 1990s, ..., those innovations began to offer sharply higher prospective returns on investment than had prevailed in earlier decades... By 1995, the investment boom had gathered momentum, suggesting that earlier expectations of elevated profitability had not been disappointed... Now, five years later, there can be little doubt that ... the [productivity] growth rate has continued to rise with scant evidence that it is about to crest." (Greenspan 2000: 2)

Die These wurde durch den ungewöhnlichen Konjunkturverlauf der USamerikanischen Wirtschaft anscheinend bestätigt. Wie den Schaubildern 1-4 entnommen werden kann, unterschied sich der Clinton-Aufschwung ab seinem fünften Jahr signifikant von vorhergehenden Aufschwüngen: die Produktivität stieg weiter an, die Inflation nahm ab, die Gewinne gingen nur geringfügig zurück und die Reallöhne stiegen.

Einer der Gründe für die außergewöhnliche Produktivitätssteigerung in einer späten Phase des Konjunkturaufschwungs war der rasche Anstieg der informationstechnologischen Kapitalintensität, insbesondere der Ausrüstungen für die Datenverarbeitung. Der in EDV-Ausrüstungen ausgelegte Teil des Kapital- 
stocks pro Arbeitsstunde stieg in der Periode von 1991-95 um durchschnittliche 16,3\% pro Jahr an und beschleunigte sich in der Periode 1996-99 auf 33,7\%. Der Einsatz von Software nahm ebenfalls rasch zu, wenngleich auch nicht ganz so dramatisch. Alle anderen Formen des Kapitalstocks, die immerhin mehr als 95\% des gesamten US-amerikanischen Kapitalstocks ausmachen, stiegen in der neunziger Jahren lediglich um ein halbes Prozent pro Jahr an (siehe Schaubild 5). Eine Studie von Oliner und Sichel ergab, dass in den Jahren 1996-99 die Investitionen in Computerausrüstungen zu 24\% zur allgemeinen Arbeitsproduktivitätssteigerung beigetragen haben, was um so erstaunlicher sei, als der Anteil von Computern am gesamten Kapitalstock 1998 geringer als 1\% gewesen sei (Oliner/Sichel 1999, 2000). Insgesamt soll die verstärkte Verwendung von Informationstechnologien (Computern, Software und Kommunikationsausrüstungen) in der zweiten Hälfte der neunziger Jahre, je nach Studie, zwischen 48\% und 74\% der Beschleunigung der Produktivitätssteigerungen von 1,06 Prozentpunkten erklären können (DOC 2000: 38).

Wie der US-amerikanische ökonomische Mainstream den langen Konjunkturaufschwung theoretisch zu fassen versucht, hat Barry Bluestone - mit kritischer Absicht - sehr anschaulich dargestellt (1999). In dieser Erklärung spielt die Inflation eine zentrale Rolle. Falls Preissteigerungen zu erwarten sind, werden Anleger als Ausgleich für die erwarteten Kaufkraftverluste höhere Zinsen verlangen. Unsicherheit über die künftige Inflationsentwicklung hemmt ebenso die Investitionstätigkeit. Ohne die Kenntnis künftiger Preise fällt es den Unternehmen schwer, das künftige Produktionsniveau zu planen. Sie werden insbesondere aus Angst vor unausgelasteten Kapazitäten langfristige Investitionen vermeiden wollen.

Die einzelnen Bestandteile des neoklassischen Wachstumsmodells fasste Bluestone wie folgt als Syllogismus zusammen:

(A) Wirtschaftliches Wachstum kann nur durch höhere Investitionen beschleunigt werden.

(B) Höhere Investitionen bedürfen niedriger Zinsen.

(C) Niedrige Zinsen bedürfen stabiler Preise und vermehrten Sparens.

(D) Nur wenn Inflationstendenzen unter strikter Kontrolle bleiben und eine hohe Sparquote erreicht wird, kann das wirtschaftliche Wachstum beschleunigt werden.

Diese logische Schlusskette lässt jedoch eine zentrale Frage offen, nämlich die nach der Nachfrageseite. Wenn durch die derart geförderten Investitionen das Produktionsvolumen ständig steigt, aber die Nachfrage für die zusätzlichen Waren und Dienstleistungen nicht ausreicht, dann erhöhen die Produktivitätssteigerungen allein die Arbeitslosigkeit und führen zu einer Unterauslastung der Produktionskapazitäten.

Diese Argumentationslücke füllt die von Bluestone so titulierte Wall Street Wachstumsspirale. Ihr liegt die Annahme zugrunde, dass Inflationsbekämp- 
fung ein Gefühl von Finanzmarktstabilität vermittelt, das selbst wiederum höhere Aktienkurse begünstigt. Diese höheren Aktienkurse vermehren das Vermögen der Haushalte und ermutigen sie dadurch zu höheren Ausgaben. Auf diese Weise entsteht die Nachfrage für das durch die neuen Investitionen zusätzlich entstandene Produktionsvolumen. Und so dreht sich die Wachstumsspirale auf ein immer höheres Niveau.

Aus heutiger Sicht scheint dieses Modell - so schreibt Bluestone 1999 - genauso funktioniert zu haben, wie es angepriesen wurde. Die Kurse des DowJones 30 Industrials stiegen von weniger als 3.800 im Jahre 1994 auf über 9.000 im Sommer 1998. Diese Kursanstiege erhöhten das Vermögen der Haushalte um zwölf Billionen US-Dollar innerhalb von lediglich vier Jahren. Jedoch wurde nur ein sehr geringer Teil dieses Vermögenszuwachses tatsächlich für Waren und Dienstleistungen ausgegeben. Doch selbst wenn dieser Teil nicht größer als 4\% gewesen wäre, hätte er die jährlichen Konsumausgaben um 120 Mrd. US-Dollar erhöht. Dadurch wäre der Konsum insgesamt um 2,1\% pro Jahr gestiegen. Wiederum in den Worten von Alan Greenspan:

„... our economy is still enjoying a virtuous cycle, in which, in the context of subdued inflation and generally supportive credit conditions, rising equity values are providing impetus for spending and, in turn, the expansion of output, employment, and productivity-enhancing capital investment." (Greenspan 1998)

Bluestones Kritik setzt an den einzelnen Bestandteilen des neoklassischen Syllogismus an, wobei nicht zuletzt seine Skepsis hinsichtlich der Steigerungsfähigkeit der Aktienkurse im Börsenjahr 2000 nachdrücklich bestätigt wurde. Gleichwohl unterscheidet er sich selbst von dieser neoklassischen Erklärung für den ungewöhnlichen Konjunkturverlauf der USA im wesentlichen nur durch die Betonung des Beitrages der informationstechnischen Revolution zur gestiegenen Investitionsbereitschaft der Unternehmen. Und mit dieser Betonung findet er sich in der Gemeinschaft mit Greenspan wieder.

\section{Die Überwindung des Computer-Paradox?}

Trotz des imposanten Konjunkturverlaufs der letzten Jahre findet die These von einer New Economy nicht unter allen Ökonomen Anhänger. Zum einen sehen einige Makroökonomen die höheren Produktivitätsfortschritte umgekehrt als Folge des hohen Wirtschaftswachstums (Solow, zitiert in Uchitelle 2000a), und dieses führen sie vor allem auf eine wachstumsfördernde Geldpolitik zurück (siehe unten). Zum anderen wird die gegenläufige Produktivitätsentwicklung entweder gänzlich angezweifelt oder als kurzzeitiger „Ausreißer“ interpretiert. Die durchschnittliche Produktivitätssteigerung von 2,15\% pro Jahr im Zeitraum vom 4. Quartal 1995 bis zum 1. Quartal 1999 lag, wie Robert Gordon hervorhob (1999a), immer noch unter der durchschnittlichen Zuwachsrate von 2,63\% im Zeitraum von 1952 bis 1972. Allerdings brachte 
die weitere Entwicklung des Jahres 1999 die durchschnittliche Produktivitätssteigerung auf 2,82\% (4.Quartal 1995 - 4. Quartal 1999; Gordon 2000: Tab. 2). ${ }^{3}$ Für die Skeptiker gilt das Diktum von Robert Solow: „You can see the computer age everywhere but in the productivity statistics" (1987: 36). Bis vor kurzem bestand nämlich unter den Produktivitätsexperten der Konsens, dass die Informationstechnologien ein Produktivitätsparadox aufwerfen würden. Die Leistungsfähigkeit dieser Technologien verbessert sich rasch, die Produktivität ihrer Herstellung ebenso und seit Beginn der achtziger Jahre finden sie eine weite Verbreitung, doch in den makroökonomischen Produktivitätsstatistiken fände ihr Einsatz keinen Niederschlag (Blinder/Quandt 1997). Auch noch im Jahre 2000 warnen führende Produktivitätsexperten wie Dale Jorgenson and Kevin Stiroh vor zu vorschnellen Urteilen:

„The apparent combination of slow productivity growth and heavy computer-use [in specific service industries] remains an important obstacle for new economy proponents who argue that the use of information technology is fundamentally changing business practices and raising productivity throughout the U.S. economy." (Jorgenson/Stiroh 2000: 37)

Des weiteren erscheint es diesen Autoren wenig plausibel, dass der relativ kleine Sektor Informationstechnologien (siehe oben) zu Produktivitätsfortschritten auf breiter Basis geführt hätte. Gemäß den Berechnungen von Robert Gordon beschränkten sich hohe Produktivitätszuwächse vornehmlich auf diesen Sektor selbst, nämlich jährlich 41,7\% vom 4. Quartal 1995 bis zum 1. Quartal 1999. Für Investitionsgüter und langlebige Konsumgüter $(d u-$ rable goods) ohne Computer lag die Produktivitätssteigerung im selben Zeitraum nur bei $1,8 \%$, beim großen Rest der Wirtschaft (nonfarm, nondurables private business sector) lediglich bei 1,5\% (Gordon 1999a: Tab. 1). Da dieser Rest hohe Computer-Investitionen getätigt hat, bleibt für Gordon das Produktivitätsparadox bestehen. Allerdings hält er das Paradox für durchaus erklärbar. Zum einen sei die Erfindung des Computers und des Internets weit weniger bedeutsam als die zentralen Erfindungen der zweiten industriellen Revolution im Zeitraum von 1860 bis 1900 (Elektrizität, Verbrennungsmotor, Kommunikationsmedien etc.). Der Computer wecke kaum neue Bedürfnisse, sondern diene vornehmlich der Rationalisierung. Doch in vielen Bereichen könne der Computer menschliche Arbeitskraft nicht ersetzen, insbesondere dort nicht, wo der persönliche Kontakt wichtig ist. Die Computernachfrage erhöhe sich deshalb nur aufgrund sinkender Preise (konstante Nachfragekurve). Auch im Zeitalter des Internets hätte sich diese Nachfrage nicht im Verhältnis zum Preisverfall erhöht. Mithin tauge die Computerrevolution nur

3 Die im September 2000 vom Bureau of Labor Statistik herausgegebene Produktivitätsstatistik auf der Basis der revidierten National Income and Product Accounts weist für den Zeitraum 1995 - 1998 ein jährliches Produktivitätswachstum von 2,5\% in der privaten Wirtschaft auf (pro Stunde aller Beschäftigten), für den Zeitraum von 1948 - 1973 eine Steigerung von 3,3 \% (BLS Multifactor Productivity Trends, 1998). 
begrenzt als gesamtwirtschaftlicher Wachstumsmotor (Gordon 2000: 24-28). Zum anderen bezweifelt Gordon, dass die Computerrevolution sogenannte Spillover-Effekte vorweisen kann. Außerhalb des verarbeitenden Gewerbes hätte selbst das Internet nur einen geringen Beitrag zur Produktivitätssteigerungen geleistet, wofür Gordon (2000: 6) fünf Gründe anführt:

- Der Gebrauch des Internets ersetzt zumeist lediglich andere Formen von Unterhaltung und Informationsbeschaffung.

- Das Internet erhöht nicht den Konsum, sondern geht ausschließlich zu Lasten bisheriger Verkaufsformen. Deshalb wird vornehmlich zur Verteidigung von Marktanteilen ins Internet investiert.

- Der Inhalt des Internets ist vielfach nicht wirklich neu, sondern macht diesen nur leichter und billiger erhältlich.

- Viele Websites verdoppeln bisherige Formen der Informationsvermittlung statt sie zu ersetzen, so dass die Kosten häufig stärker als der Umsatz steigen.

- Das Internet wird größtenteils während der Arbeitszeit auf Kosten der Unternehmen benutzt.

Robert Gordon bestreitet nicht, dass in den neunziger Jahren bereits eine geringere Arbeitslosenquote inflationsneutral blieb und mithin ein höheres Wirtschaftswachstum möglich war. Für diese Absenkung der NAIRU führt er allerdings in erster Linie ein gegenüber den achtziger Jahren verändertes Verhalten der Preise für Nahrungsmittel und Energie sowie fallende Importpreise an. Erst in zweiter Linie sei die günstigere NAIRU durch fallende Computerpreise, durch das Abflachen der Preissteigerungen im Gesundheitssektor (dank den Kürzungen der von den Unternehmen gewährten Sozialleistungen) und durch die veränderte Bemessungsmethode der Preissteigerungsrate bedingt worden (Gordon 1999b).

\section{Regulationstheoretische Deutung der New Economy}

Die These von einer New Economy wird allerdings nicht nur von interessierter Seite, sei es die Clinton-Regierung oder Emissionshäuser an der Wall Street, propagiert, sondern auch von Wirtschaftshistorikern, wie Paul David, und Gesellschaftstheoretikern aus einer neomarxistischen Tradition, wie Manuell Castells. ${ }^{4}$

Das Produktivitätsparadox der Informationstechnologien wird von diesen durch die Argumentationsfigur „neues Produktionsparadigma“ gelöst: Derzeit wäre der Durchbruch eines neuen Produktionsparadigmas beobachtbar. Die Produktion mittels Technologien auf Basis billiger Energien würde durch ein Produktionsparadigma auf Basis billiger Informationen abgelöst werden. Die-

4 Manuel Castells Definition der New Economy bezieht sich allerdings weniger auf ihre makroökonomische Dimension als vielmehr auf ihre Produktionsseite. Für ihn ist die New Economy ,informational, global, and networked“. 
ser Wechsel sei durch die informationstechnologische Revolution ermöglicht worden. Verbindendes Element der verschiedensten technologischen Durchbrüche der letzten Jahrzehnte sei die gemeinsame digitale Sprache für die Beschaffung, Speicherung und Übertragung von Informationen. Die Digitalisierung von Informationen begünstige die Möglichkeiten des Austausches zwischen einzelnen Forschungsfeldern und beschleunige somit die Wissensgenerierung. Allerdings könnten die Früchte dieser technologischen Entwicklung nicht sofort gesamtwirtschaftlich geerntet werden. In Analogie zu technologischen Sprüngen in der Vergangenheit (z.B. Erfindung und Verbreitung des Elektromotors; David/Wright 1999), müsse von einer längeren Lernphase im Umgang mit der neuen Technik ausgegangen werden. Der entscheidende Durchbruch für die allgemeine Anwendung der elektronischen Datenverarbeitung sei erst 1971 mit der Erfindung des Mikroprozessors vollzogen worden. Sein Einsatz ging allerdings mit hohen Lernkosten einher. Es bedurfte der Veränderung des institutionellen Umfeldes. Studien zur Einführung von elektronischer Datenverarbeitung in Organisationen ergaben, dass sich die Produktivitätsgewinne nur dann einstellten, wenn die Organisationen entsprechend umstrukturiert wurden (Brynjolfsson/Hitt 1998). Die optimale Nutzung des Computers sei erst mit der Entstehung des Internets gegeben gewesen (Castells 2000: 51-53). Eigene Erfahrungen im Umgang mit dem Computer in der Wissenschaft dürften dieses Argument rasch plausibel erscheinen lassen.

Aber nicht nur dass die elektronische Datenverarbeitung erst im Laufe der Zeit ihr volles Potenzial entfalten konnte, führte zu der unerwartet enttäuschenden Produktivitätsentwicklung. Von größerer Bedeutung dürfte die Ablösung des Alten durch das neue Paradigma gewesen sein. Folgt man der Regulationstheorie in der Annahme, dass die Produktivitätsreserven des alten Paradigmas spätestens seit Mitte der siebziger Jahren erschöpft gewesen seien (Aglietta 1979: 119-121), dann waren die geringen Produktivitätssteigerungen in den achtziger und frühen neunziger Jahren geradezu zwangsläufig. Denn solange das neue Paradigma nur in wenigen Bereichen zur Anwendung kam und in einer noch geringeren Anzahl von Bereichen die erhoffte Wirkung zeigte, prägte die abnehmende Effizienz des alten Paradigmas die gesamtgesellschaftliche Produktivitätsentwicklung (David 1999: 29).

Laut der Regulationstheorie kann ein neues Produktionsparadigma sein Potenzial jedoch nicht voll entfalten, solange es nicht in ein kompatibles Akkumlationsregime und eine entsprechende Regulationsweise eingebettet ist (Aglietta 1979). Im folgenden will ich ein Modell eines New-EconomyAkkumlationsregimes auf der Basis des informationstechnischen Produktionsparadigmas skizzieren, dass sich an ein von Robert Boyer entworfenes „finanzmarkt-getriebenes" Wachstumsmodell (2000) anlehnt. Mit Beispielen aus den USA soll dieses Modell veranschaulicht werden. 
Gegenüber dem Akkumulationsregime des Fordismus, in dem Investitionen vornehmlich vom Konsum angetrieben werden, käme in der New Economy diese Rolle den Erwartungen in die Effizienz-, Pionier- und Monopolgewinne bei der Anwendung von technologischen Innovationen zu. Ausreichend gesellschaftliche Nachfrage bliebe erstens durch die beschäftigungswirksamen Multiplikatoreneffekte der Investitionen gesichert. Zweitens würde das Reallohnniveau im Gegensatz zum Fordismus nicht durch eine Anpassung der Nominallöhne an das Produktivitätswachstum gehalten werden, sondern vornehmlich durch Preissenkungen. In der vor-fordistischen Periode behinderte eine solche Strategie die Integration der Masse der Lohnabhängigen in die kapitalistische Konsumwelt. Zentrale Konsumprodukte, wie das Automobil und das Eigenheim, konnten vor der Weltwirtschaftskrise noch so billig werden, sie blieben absolut weiterhin so teuer, dass sie nicht aus laufenden Einkommensströmen finanziert werden konnten. Die Masse der Lohnabhängigen hatte zudem aufgrund prekärer Einkommensströme kaum Zugang zu Kredit. Heute fällt in den OECD-Ländern die soziale Absicherung trotz Umbau des Sozialstaates immer noch deutlich umfangreicher aus, sodass die Einkommensströme der Lohnabhängigen vergleichsweise stetig fließen (ausgenommen die von der US-Sozialhilfereform Betroffenen; Piven 1999, Hay 2001). Nicht zuletzt aus diesem Grund sind heute Kredite (bzw. Kreditkarten) für die Masse der Lohnabhängigen leicht zugänglich. Ferner werden die Preise für einfache Konsumgüter durch Importe aus Ländern mit deutlich geringerem Lohnniveau niedrig gehalten, was vor allem für die Befriedigung der Konsumbedürfnisse der unteren Lohngruppen bedeutsam ist. Allerdings erlauben die hohen Produktivitätszuwächse durchaus auch inflationsneutrale Nominallohnsteigerungen, wie sie in den letzten drei Jahren in den USA beobachtbar waren (siehe Schaubild 4; die realen Stundenlöhne stiegen von 1995-1998 um durchschnittlich 1,9\% pro Jahr, wobei die unteren Lohngruppen nicht zuletzt aufgrund der Erhöhung des Mindestlohns deutlich höhere Steigerungen verzeichnen konnten, Mishel et al. 2000: 115, 124). Drittens wird durch die gewinninduzierte Vermögensmehrung die Konsumneigung begünstigt. Steigende Immobilienpreise und Aktienkurse ermutigten die Haushalte zu höheren Konsumausgaben (vergleichbar mit dem Argument von der Wall Street Wachstumsspirale; siehe oben).

Die Regulation des Lohnverhältnisses würde in der New Economy weitgehend marktförmig ausfallen. Auf der Basis eines staatlich vorgegebenen Mindestniveaus an Lohnhöhe und sozialer Absicherung würden sich Preis und Menge der Arbeitskraft nach Angebot und Nachfrage richten. Die daraus resultierende Lohnspreizung würde beschäftigungsfördernd wirken. Geringe Löhne bei Wirtschaftsaktivitäten mit geringer Produktivität erlaubten deren Aufrechterhaltung bzw. erlaubten es den Besserverdienenden derzeit noch im Haushalt durch unbezahlte Arbeit erbrachte Dienstleistungen über den 
Markt zu beziehen (Häußermann 1999). Aus den oben genannten Gründen sei trotz der Lohnspreizung keine Nachfragelücke zu befürchten. Ein wachsender Anteil der Entlohnung würde ferner gewinnabhängig ausgezahlt, sei es in Form von Bonuszahlungen oder von Aktienoptionen (siehe dazu auch Priewe in diesem Heft). Darüber hinaus würden Rückstellungen für die Altersversorgung zunehmend kapitalisiert, sei es in Form privater Altersvorsorge oder betrieblicher Pensionskassen, die ihre Gelder frei auf den Vermögensmärkten anlegen können. Dadurch würden die Beschäftigten an den Gewinnen und an der durch diese verursachten Vermögensvermehrung beteiligt werden. $\mathrm{Da}$ in diesem Modell Investitionen vornehmlich von Gewinnen abhängig sind, können die Beschäftigten durch Lohnzurückhaltung die Gewinne und damit auch das Beschäftigungsniveau günstig beeinflussen und zugleich ihre Vermögensposition verbessern. Zudem bestünde ein direkter Anreiz, produktiver zu arbeiten.

Weitgehend deregulierte, globalisierte Märkte böten eine Gewähr gegen Inflationstendenzen und führten zur raschen, innovationsfördernden Reallokation von Ressourcen. Vor allem die Finanzmärkte würden rascher auf neuen Bedarf reagieren und somit Innovationen anschieben und Strukturkonservatismus verhindern. Statt Selbstfinanzierung und Bankkredite würden börsennotierte Wertpapiere und Venture-Kapital als Finanzierungsarten dominieren. Dies hätte den zusätzlichen Nutzen der Nachfragestabilisierung, denn steigende Aktienkurse führen zu erhöhten Konsumausgaben. Allein die Geldpolitik bliebe wie im Fordismus staatlich verfasst, zumindest in den industriell führenden Kernlanden.

\section{Unsichere Kausalitäten im New-Economy-Modell}

Ähnlich dem neoklassischen Syllogismus scheint dieses regulationstheoretisch inspirierte Modell der New Economy das dynamische Wirtschaftswachstum der USA in den letzten Jahren auf den ersten Blick plausibel erklären zu können. Eine regulationstheoretische Deutung beinhaltet jedoch zugleich auch das Aufspüren von Zusammenhängen, deren Reproduktion nicht automatisch gesichert ist. Im Modell bestehen mindestens drei Konstellationen, für die der unterstellte Kausalzusammenhang nicht per se gegeben ist.

Der Investitionen-Gewinn-Nexus: Das Modell geht davon aus, dass Investitionen in die Ausrüstungsgüter des neuen Produktionsparadigmas sowohl die Produktivität als auch die Gewinne erhöhten. Die Produktivitätszuwächse erlauben das höhere Wirtschaftswachstum und die Gewinnaussichten veranlassen die Investitionen. Dass sich die erwarteten Gewinne auch einstellen, ist allerdings aus mehreren Gründen nicht automatisch gesichert. Erstens weist sich die New Economy gerade dadurch aus, dass sie sich an der technologischen Innovationsfront bewegt. Deshalb besteht große Unsicherheit darüber, 
inwieweit sich die jeweils ausgesuchte Technik bewähren wird. Fehlinvestitionen sind deshalb kaum vermeidbar (Leonhardt 2000). Aus einem systematischen Grund ist zudem anzunehmen, dass im Produktionsparadigma der New-Economy solche Fehlinvestitionen einen beträchtlichen Umfang annehmen können. Da sich das neue Produktionsparadigma nicht zuletzt durch Netzwerkexternalitäten auszeichnet (d.h. die Vorteile wachsen mit der Zahl der Nutzer eines Netzwerks), winkt dem Unternehmen besonderer Gewinn, das den Standard für das Netzwerk setzen kann. Aufgrund dieser Gewinnanreize werden sehr viele Unternehmen versuchen, im jeweiligen Netzwerk den Standard zu setzen. Da letztlich nur ein Unternehmen gewinnen kann, werden die Investitionen der anderen Unternehmen notgedrungen ohne Ertrag bleiben (der sogenannte industry shake-out), es sei denn, die Ausrüstung bzw. der Kundenstamm kann vom Gewinner übernommen werden. Ferner können hohe Gewinnerwartungen dazu führen, dass Inhaber von strategischen Ressourcen zur Ausnutzung von Netzeffekten noch nicht realisierte Gewinne abschöpfen (Beispiel: die staatlichen Auktionen der UMTS-Lizenzen im Mobilfunkverkehr). Zweitens gehört zur Kehrseite der informationstechnologischen Revolution, dass sie die Nachahmung von Innovationen erleichtert (Leonhardt 2000). Das Nachahmen führt zwar zur rascheren Verbreitung der Produktivitätseffekte, und ist somit volkswirtschaftlich wünschenswert, aber es mindert die Pioniergewinne. Drittens kann sich die im Modell angestrebte inflationsbekämpfende Wechselkurspolitik negativ auf die Gewinnentwicklung auswirken, indem sie durch eine Aufwertung der eigenen Währung die Produkte der ausländischen Konkurrenz verbilligt und zwar nicht nur deren einfache sondern auch deren komplexe Produkte (für das Jahr 2000 wird mit dem Verschwinden des traditionellen Überschusses im Handel mit Hi-Tech-Gütern gerechnet; Lenz 2000: 5-2). Das Ziel der Preisstabilität kann somit in Konflikt mit dem Gewinnziel geraten. Wenn aus dem Zusammenspiel dieser drei Gründe die Gewinnerwartungen nach einer gewissen Zeit nicht eintreffen, dann käme es in diesem Modell zu einem Rückgang der Investitionen. Ein Konjunktureinbruch wäre somit nicht auszuschließen. Aufgrund der Bedeutung, die der Vermögensvermehrung in diesem Modell zukommt, wäre in einem solchen Fall sogar mit einem zyklischen Verstärkungseffekt zu rechnen.

Der Vermögen-Gewinn-Nexus: Das New-Economy-Modell unterstellt, dass die an der Börse vorgenommene Bewertung von Anteilsscheinen am Produktivvermögen in einem engen Zusammenhang mit den Gewinnerwartungen der betreffenden Unternehmen steht. Steigende Gewinne führten zu einer Höherbewertung an der Börse und somit zu einem Vermögenszuwachs bei den Anlegern. Langfristig mag dies zutreffen, doch wie unzählige Studien aufzeigen, gehört die Hoffnung auf Kursgewinne und nicht nur auf Dividenden zu den wesentlichen Motiven zur Teilnahme an der Börse (zuletzt Shiller 2000). 
Deshalb fließt in die Anlageentscheidung nicht nur eine Einschätzung der zu erwartenden künftigen Gewinne sondern auch eine Abschätzung der Gewinneinschätzungen der anderen Marktteilnehmer ein. Aus einzelnen rationalen Entscheidungen kann es deshalb zu einem in Bezug auf die Ertragsstärke der Anlageobjekte irrationalen Herdenverhalten der Anleger kommen (vgl. zu dem selbst von Alan Greenspan als irrational exuberance bezeichneten Börsenfieber der letzten Jahre Evans im vorliegenden Heft). Solche Börsenspekulationen können im Zeitalter globaler Finanzmärkte durch Wechselkursspekulationen noch verstärkt werden, da für währungsfremde Anleger die Ertragsstärke eines Anlageobjektes im Verhältnis zu den Wechselkursschwankungen eine unerhebliche Größe sein kann. (Mit der Abwertung des Yen ab 1995 und auf der Suche nach sicheren Anlagemöglichkeiten im Gefolge der Asienkrise strömten ausländische Anleger an die US-Börsen, wo sie im 1. Halbjahr 2000 ca. 30\% aller Anteilsscheine kauften; Brenner 2000: 28). Mithin muss aus systematischen Gründen mit zyklischen Schwankungen der marktförmigen Vermögensbewertung gerechnet werden, die sich im Falle einer Baisse insbesondere auf den Vermögen-Konsum-Nexus negativ auswirken.

Der Vermögen-Konsum-Nexus: Gleichfalls ist die Annahme, dass Vermögenszuwächse die Konsumneigung erhöhen, sehr voraussetzungsvoll. Bereits in der Auseinandersetzung mit dem neoklassischen Wachstumssyllogismus hat Bluestone darauf hingewiesen, dass steigende Aktienkurse keine realen Einkommensströme schaffen, sondern lediglich die Kurswerte erhöhen. Vielmehr würden sich steigende Aktienkurse auf die Güterwirtschaft im wesentlichen nur psychologisch auswirken. Es sei nur dann mit nennenswerten psychologischen Auswirkungen zurechnen, wenn die Kurssteigerungen außerordentlich hoch ausfallen und sie mehr oder weniger als dauerhaft angesehen werden würden. Zudem wird das Vermögen überproportional von den reichsten Haushalten besessen, die zu sehr hohen Sparquoten neigen. Zur Erhöhung des Konsumniveaus bedürfte es deshalb gewaltiger und kontinuierlicher Steigerungen der Aktienkurse (1999: 34). Die obige Problematisierung des Investitions-Gewinn-Nexus stellt solche Steigerungen selbst unter der Annahme eines funktionierenden Vermögen-Gewinn-Nexus in Frage.

Die Kontingenzen dieser drei Zusammenhänge lassen zyklische Schwankungen im Modell der New Economy wahrscheinlich sein. Im Jahre 2000 kam es tatsächlich in allen drei dieser Zusammenhänge zu Störungen. Im Investitionen-Gewinn-Nexus enttäuschten nicht nur die so genannten „dot.com“ Firmen die Gewinnerwartungen der Finanzanalysten sondern auch ein breites Spektrum der börsennotierten Unternehmen. Für die Standard \& Poor's 500 Firmen mussten die Gewinnerwartungen für das 4. Quartal 2000 von 15,4 auf 4,2\% gekürzt werden (Fuerbringer 2001). Im Vermögen-Gewinn-Nexus drohten die Kurse nun umgekehrt ins Bodenlose zu fallen (von September bis Ende Dezember fielen die Kurse der Technologiebörse NASDAQ um 45\%). 
Die dadurch ausgelösten Kursverluste bremsten die Konsumneigung, und zwar vor allem der vermögenderen Haushalte (Uchitelle 2000b).

Zum Teil wurden diese konjunkturbremsenden Entwicklungen durch die ab Mitte 1999 vorgenommenen Zinserhöhungen der Zentralbank ausgelöst. Die Erhöhung der Zinsrate auf 6,5\% erfolgte angesichts neuer Inflationsgefahren, die einerseits dem raschen, die Produktivitätssteigerungen übertreffenden Wirtschaftswachstum und zum anderen der begrenzten Elastizität der Rohstoffmärkte geschuldet waren (FRB 1999; Ölpreise verdoppelten sich von Mitte 1999 bis Mitte 2000, BOC 2000). Diese Entwicklungen weisen darauf hin, dass in der New Economy aufgrund der irrational exuberance der Börsen, die Gefahr einer konjunkturellen Überhitzung nicht gebannt ist. Ferner zeigte es sich, dass auch die New Economy trotz der informationstechnologischen Revolution von der billigen Zufuhr von Rohstoffen abhängig ist.

Konjunkturzyklen stellen allerdings ein Wachstumsmodell nicht grundsätzlich in Frage, auch im „goldenen“ Fordismus machten sie sich bemerkbar. Die Frage ist vielmehr, ob das Wachstumsmodell über automatische oder zumindest über diskretionäre Stabilisatoren verfügt. Die Steuerungsaufgabe schreibt Robert Boyer in seinem finanzmarkt-getriebenen Wachstumsmodell der Zentralbank zu, die zusätzlich zur Inflationsbekämpfung noch die Börsenkurse durch Zinspolitik und Liquiditätsbereitstellung verstetigen muss (2000: 131f.). Für das hier auf der Basis eines neuen Produktionsparadigmas entwickelte Wachstumsmodell kommt die Funktion der Verstetigung des Investitionsvolumens hinzu. Im Falle umfangreicher Fehlinvestitionen müsste die Zentralbank verunsicherten Investoren durch Zinssenkungen einen Anreiz geben, weitere Investitionsrisiken einzugehen. Im Abschwung müsste die Zentralbank auch Deflationstendenzen entgegensteuern, die aufgrund der fehlenden tarifvertraglichen Anbindung der Nominallöhne an das Produktivitätswachstum zu entstehen drohen.

\section{Dünne empirische Basis zur Beurteilung der New-Economy-These}

Die zentrale Annahme dieser regulationstheoretischen Deutung der New Economy, nämlich hohe Produktivitätszuwächse aufgrund eines neuen Produktionsparadigmas, steht allerdings auf einer fragwürdigen empirischen $\mathrm{Ba}-$ sis. Wie bereits erwähnt, weisen die amtlichen Statistiken kein signifikant beschleunigtes Wachstum außerhalb der informationstechnologischen Industrien aus. Für Dienstleistungsbranchen, die zwischen 1990 und 1997 im besonderen Maße Informationstechniken eingesetzt haben, ergab eine Untersuchung des Wirtschaftsministeriums sogar, dass die Arbeitsproduktivität um 0,3\% zurückging (DOC 2000: 39f). Laut dem Starjournalisten Bob Woodward war sich Alan Greenspan bei der Formulierung der New-EconomyThese dieser Datenlage bewusst. Aufgrund einer Anomalie im Verhältnis von 
Preis- zu Gewinnentwicklung kam Greenspan zu dem Schluss, dass diese Statistiken die Produktivitätsentwicklung zu gering ausweisen. In den Jahren 1994 und 1995 stiegen nämlich die Unternehmensgewinne rasch an, wobei das Inflations- und Lohnniveau stabil blieben. Diese Differenz konnte er sich nur durch eine gestiegene Produktivität erklären (Woodward 2000: 173).

In der Tat ist die Aussagekraft der amtlichen Statistiken beschränkt. Mittlerweile tragen die Dienstleistungen zu weit mehr als der Hälfte zum Bruttoinlandsprodukt der USA bei. Für die Dienstleistungen sind jedoch noch keine allseits akzeptierten Methoden zur Messung der Produktivität entwickelt worden. Wiederum weit über die Hälfte der Dienstleistungen gelten als kaum messbar. Zu diesen gehören das Erziehungswesen, das Gesundheitswesen und der große Bereich FIRE (Finanzdienstleistungen, Versicherung und Immobilienbetreuung; Griliches 1995). Wenn die Statistik den Umsatz als OutputMaßgröße wählen würde, dann würde sie beispielsweise im Falle geringerer Abschlussgebühren für Versicherungen von einer fallenden Produktivität ausgehen, wenngleich die Senkung der Gebühren der gestiegenen Produktivität der Verwaltung geschuldet sein mag. Aus diesem Grunde nimmt das USamerikanische Bundesamt für Statistik für die meisten dieser Bereiche die geleisteten Arbeitsstunden als Maßgröße für die Ausbringungsmengen. Dies führt allerdings dazu, dass das Produktivitätswachstum weitgehend mit den Lohnsteigerungen gleichgesetzt wird (Dean 1999). Ferner kann der Einsatz von Informationstechnologie die Effizienz unbezahlter anstelle von bezahlter Arbeit erhöhen (z.B. schnellere Recherchemöglichkeiten für Bibliotheksbenutzer), die sich nicht in den Output-Zahlen niederschlagen (Huws 2000: 655).

Im Oktober 1999 wechselte das Statistikamt des Arbeitsministeriums seine Methode der Produktivitätsmessung von Finanzdienstleistungen. Deren Transaktionen wurden nunmehr als Maß des Outputs genommen, wodurch hohe Arbeitsproduktivitätszuwächse sichtbar wurden. Allerdings stellt sich diesbezüglich die Frage der Gewichtung der unterschiedlichen Transaktionen, die von Kontobuchungen bis zu komplexen Beratungsleistungen reichen (DOC 2000: 69). Nicht zuletzt auf Initiative von Alan Greenspan beschäftigte sich eine Vielzahl von Studien mit dem Problem der Produktivitätsbemessung in einzelnen Dienstleistungsbranchen. Sie bestätigten zumeist Messfehler, die zu einer Unterschätzung der realen Produktivitätsentwicklung führten. Über das Ausmaß dieser Unterschätzung besteht allerdings kein Konsens (Dean 1999).

Die Messung der Produktivität im güterproduzierenden Sektor erfolgt allerdings auch nicht ohne Schwierigkeiten, denn es ist prinzipiell problematisch wenn eine physische Größe (Arbeitsstunden) zu einer monetären Größe (Wertschöpfung) ins Verhältnis gesetzt wird. So wird beispielsweise der Nutzen einer gestiegenen Produktvielfalt (die aufgrund geringerer Skalenerträge 
produktivitätshemmend wirkt) ebenso wenig wie Verbesserungen am Produkt adäquat berücksichtigt. Allerdings lässt sich beim Versuch, qualitative Verbesserung zu berücksichtigen, Willkür nicht vermeiden. So hat das Amt für Wirtschaftsstatistik einen „hedonistischen“ Preisindex für die Computer- und Halbleiterindustrie entwickelt, der die Veränderungen des Preis-Leistungsverhältnisses reflektiert. Doch während im Computerbereich die höhere Rechengeschwindigkeit in die Ermittlung der Arbeitsproduktivität eingeht, wird beispielsweise im Fahrzeugbau nicht die maximale Geschwindigkeit der hergestellten Fahrzeuge berücksichtigt. Die Entwicklung eines „hedonistischen“ Preisindizes läuft deshalb Gefahr, interessengeleitet zu erfolgen. Interessanterweise wurde der Preisindex für die Computerindustrie gemeinsam mit einem Team von IBM entwickelt (Cole et al. 1986).

Desweiteren ist noch ungeklärt, ob beispielsweise die in betrieblichen Kostenvergleichsstudien ermittelten Produktivitätssprünge in der Auto- und Stahlindustrie tatsächlich auf den Einsatz von Informationstechniken zurückgeführt werden können. Der signifikante Produktivitätsvorsprung der japanischen Hersteller in den 1980er Jahre beruhte nicht auf einem höheren Anteil von Informationstechniken (es sei denn man rechnet das auf Karteikärtchen beruhende kanban-System hinzu), sondern auf den mittlerweile allseits bekannten Prinzipien der schlanken Produktion und einer hohen Kapazitätsauslastung (Jürgens et al. 1989; Scherrer 1992). Aus dieser Sicht wäre die positive Produktivitätsentwicklung der Old Economy Folge eines Lernens von Japan, und zwar einerseits hinsichtlich der Technik und andererseits dank des günstigen makroökonomischen Klimas auch bezogen auf höhere Kapazitätsauslastungsgrade. Zudem werden mittlerweile 30\% der in den USA hergestellten PKWs unter der Regie japanischer Manager produziert.

Angesichts dieser Bemessungsprobleme kam das Wirtschaftsministerium in seiner Studie „Digital Economy“ trotz der insgesamt positiven Bewertung der New-Economy-These zu der vorsichtigen Einschätzung:

„In the absence of more accurate measures of output for IT-intensive services industries, we cannot rule out the possibility that IT has made a very modest contribution to labor productivity outside the IT producing sector itself." (DOC 2000: 69)

Die Kritik an der New-Economy-These steht allerdings auf der gleichen dünnen empirischen Basis. Robert Gordons Versuch, diese These empirisch zu widerlegen, basiert auf den mit Messfehlern behafteten amtlichen Produktivitätsstatistiken. Darüber hinaus überzeugt sein Vorgehen bei der Widerlegung der These eines Spillover-Effektes nicht. In seiner regressionsanalytischen Untersuchung des Zeitraumes von 1994 bis 1999 bedarf es zur Erklärung der Produktivitätszuwächse in der privaten Wirtschaft einschließlich der Computerbranche eines konjunkturellen Effektes der über den bisherigen Trend hinausgeht. Zur Erklärung des Produktivitätswachstums in der privaten Wirtschaft außerhalb der Computerindustrie reicht allerdings der Trend aus. Dar- 
aus schließt Gordon, dass der Einsatz von Computern außerhalb der Computerindustrie zu keinen Produktivitätsschüben oberhalb des Trends geführt hätte (Gordon 2000: 16). Unabhängig von der bereits problematisierten Messung des Produktivitätszuwachses bleibt die Schlussfolgerung von Gordon insofern ungesichert, als unklar ist, ob ohne den Einsatz von Computern der Trend im untersuchten Zeitraum eingehalten worden wäre. Zudem ist die Datenlage hinsichtlich der geografischen und branchenmäßigen Verteilung der Computerinvestitionen lückenhaft (Haltiwanger/Jarmin 2000: 7).

\section{Alternative Makroerklärung: Außenfinanzierter Konsumboom}

Angesichts der ungesicherten empirischen Basis der New Economy These können alternative Erklärungen ebenfalls für sich Plausibilität beanspruchen. Eine alternative Deutung findet sich bei Robert Brenner (2000), der zwar auch die Bedeutung der Produktivitätszuwächse betont, aber vor allem die zentrale Stellung deutlich gestiegener Profite herausarbeitet. Im vorliegenden Heft betont Trevor Evans vor allem die Rolle der wachstumsorientierten Geldpolitik: Seit Mitte der achtziger Jahre hat sich die US-amerikanische Geldpolitik - mit einer kurzzeitigen Ausnahme - antizyklisch verhalten. Demgegenüber sei die europäische Geldpolitik nicht nur restriktiver sondern auch prozyklisch ausgefallen. Zusammen mit einer ebenso tendenziell prozyklischen Fiskalpolitik hätte die europäische Geldpolitik zu einem deutlich höheren Zinsniveau geführt und einer entsprechend höheren Arbeitslosigkeit (vgl. Palley 1999).

Ich möchte der aus meiner Sicht sehr überzeugenden Argumentation von Evans nichts hinzufügen außer einigen Überlegungen zur Frage, inwieweit die US-Geldpolitik der neunziger Jahre verallgemeinerbar ist. In der Zeit vor der Einführung des Euro war die restriktivere Politik in Europa insbesondere eine Folge der Globalisierung wirtschaftlicher Aktivitäten. Die nationalen Währungen standen untereinander in der Konkurrenz um die Gelder der internationalen Vermögensbesitzer, niedrigere reale Zinsen wären mit einem Abzug von Geldern bestraft worden (Herr/Voy 1989). In den USA wurde hingegen die weniger restriktive Geldpolitik durch die Globalisierung der Waren- und Kapitalmärkte begünstigt. Die weitgehend liberalisierten Gütermärkte hielten die Inflation unter Kontrolle, und zwar durch einen Fall der Importpreise (vom 3. Quartal 1995 bis zum 1. Quartal 1999 um durchschnittlich 6,4\% pro Jahr, wobei dieser Fall zu 70\% von Nicht-Öl-Gütern bewirkt wurde; Rich/Rissmiller 2000: 5, Anm. 7), durch Konkurrenzdruck und durch die Bereitstellung zusätzlicher Produktionskapazitäten, durch die Enpässe vermieden wurden (ein Argument, was sich auch bei Greenspan 2000 wiederfindet). Der geringe Inflationsdruck ließ der Zentralbank den wachstumsfördernden zinspolitischen Spielraum. Die Kehrseite dieser Öffnung des eigenen Marktes 
waren allerdings wachsende Handelsbilanzdefizite, die für 2000 auf über 450 Mrd. US-Dollar geschätzt werden und somit auf etwa $4 \%$ des USamerikanischen Bruttosozialproduktes angewachsen sind (Bureau of Economic Analysis / U.S. Census, January 2, 2001). Finanziert werden diese inflationsdämpfenden Handelsbilanzdefizite mittels liberalisierter Kapitalmärkte. Im ersten Viertel des Jahres 2000 finanzierte das „Ausland“ bereits 4\% des BSP der USA (Peach/Steindel 2000: 3).

In dieser Bereitschaft ausländischer Wirtschaftsubjekte, den US-amerikanischen Aufschwung zu finanzieren, könnte die Singularität des US-Aufschwunges begründet sein. Alle anderen Länder mit samt ihren Wirtschaftssubjekten verfügen nicht über ein derart hohes Vertrauen der internationalen Geldvermögensbesitzer in ihre Zahlungsfähigkeit. Dafür bestehen trotz des Herdentriebs der Finanzmarktakteure auch objektive Gründe: das fehlende Devisenaufbringungsproblem (die Wirtschaftssubjekte der USA sind in ihrer eigenen Landeswährung verschuldet) und die größenbedingte hohe Liquidität des USMarktes (die einen raschen Marktaustritt verspricht). Allerdings, so lehrt die Vergangenheit, ist dieses Vertrauen nicht grenzenlos. In den Jahren 1971-73, 1979 und 1985 kam es zu massiven Kapitalrückflüssen, die zu drastischen Abwertungen des US-Dollars führten (Scherrer 1999: 185-234). Deshalb sind die Verantwortlichen der US-amerikanischen Geld- und Währungspolitik trotz des Bekenntnisses zur New Economy über die steigenden Leistungsbilanzdefizite beunruhigt. Eine Abkehr der internationalen Vermögensbesitzer vom US-Dollar und seine damit einhergehende Abwertung würde rasch die Importe verteuern, die Geldentwertung beschleunigen und zu harten zinspolitischen Maßnahmen drängen: Der Abschwung wäre da (Bergsten 2000).

Die Sorge wäre weniger berechtigt, wenn der Verschuldung im Ausland tatsächlich eine deutlich gesteigerte Investitionstätigkeit gegenüberstünde. Das Investitionsniveau der letzten Jahre ist in historischer Sicht nicht besonders hoch (es lag 1998 bei 16,1\% des BSP, 1980 betrug es 16,7\%) und liegt zudem unter dem europäischen Niveau (1998: 18,5\%; Pitz 2000: Chart 4). In den US-Statistiken wurden 1999 interessanterweise die Ausgaben für Computerprogramme reklassifiziert und den Investitionen zu geordnet, so dass sich das statistisch ausgewiesene Investitionsniveau deutlich erhöhte, und zwar um 149 Mrd. US-Dollar im Jahre 1999 (DOC 2000: 67). Den höheren Investitionen stehen aufgrund des raschen „moralischen Verschleißes“ der Informationstechniken zudem höhere Abschreibungen gegenüber. In den 1960er Jahren stiegen das Bruttoinlandsprodukt (BIP) und das Nettoinlandsprodukt (BIP minus den Abschreibungen) im Gleichschritt um 4,4\% pro Jahr an. 1999 wuchs das BIP um 4,1\%, während das Nettoinlandsprodukt lediglich um 3,6\% zunahm (ebd.). Da sich im Vergleich zu den 1980er Jahren das Investitionsvolumen nicht erhöht hat, finanzierte das Ausland in den letzten Jahren wohl eher den Konsum als die Investitionen. 
Die These eines konsumgetriebenen Aufschwunges trotz der hohen Investitionen in Informationstechniken ist deshalb nicht von der Hand zuweisen, zumal die Sparquote der privaten Haushalte drastisch abgenommen hat. Im August 2000 hatte sie ihren vorläufigen historischen Tiefststand von $-0,4 \%$ erreicht (Pitz 2000: 5). Richard Peach und Charles Steindel weisen zwar darauf hin, dass bei Berücksichtigung der Veräußerungsgewinne und der auf diese Gewinne gezahlten Steuern die Sparquote 1999 um 7,25 Prozentpunkte höher ausfallen würde, doch räumen sie ein, dass die Masse der Veräußerungsgewinne bei sehr wenigen Haushalten anfällt (Peach/Steindel 2000; siehe Schaubild 6). Im Jahre 2000 half dieses Gedankenspiel allerdings auch nicht weiter, da die Kursgewinne im Jahresdurchschnitt ausblieben. Im Falle eines Abflauens der Konjunktur wird auch der amerikanische Staat, der im Jahre 1999 erstaunliche 5,1\% des BSP „gespart“ hat (ebd.: 3), das mangelnde Sparen der Haushalte nicht ausgleichen können. Mithin wäre mit einer zunehmenden Abhängigkeit vom Ausland, dass 1999 bereits zu ca. 4\% das BSP finanziert hat (ebd.), zu rechnen. Eine solche Abhängigkeit führt laut Robert Brenner zu einem zinspolitischen Dilemma der Zentralbank. Zur Förderung des Konjunkturaufschwungs müsste sie die Zinsen senken, doch um den weiteren Zustrom ausländischen Geldes zu sichern, müsste sie die Zinsen anheben (Brenner 2000: 43). Derzeit scheint die Zentralbank unter Alan Greenspan zu hoffen, dass durch Zinssenkungen die Börsenkurse wieder steigen und somit die USA als Anlageland attraktiv bleiben. Die vom neuen Präsidenten George Bush geplante Steuersenkung soll ebenfalls zu diesem Ziel beitragen. Inwiefern dieses Kalkül aber langfristig aufgehen kann, hängt nicht zuletzt von den künftigen Unternehmensgewinnen ab, deren Höhe wiederum von den zukünftigen Produktivitätszuwächsen beeinflusst wird.

\section{Die New Economy bedarf noch der Bewährung}

Angesichts des beeindruckenden US-amerikanischen Konjunkturverlaufs in den letzten Jahren kommt der These von einer New Economy, die dank hoher Produktivitätszuwächse ein inflationsfreies hohes Wachstum ermöglicht, eine erhebliche Plausibilität zu. Sie lässt sich zudem sowohl innerhalb des ökonomietheoretischen Mainstream als auch regulationstheoretisch begründen. Allerdings stellte die Überprüfung der Kausalzusammenhänge im regulationstheoretischen Modell der New Economy deren zwingenden Charakter in Frage. Insbesondere kann nicht sicher davon ausgegangen werden, dass Investitionen in die Ausrüstungsgüter des neuen Produktionsparadigmas sowohl die Produktivität als auch die Gewinne erhöht. Zudem steht der Kern der New-Economy-These, die informationstechnologisch bedingten Produktivitätszuwächse, noch auf unsicherem Datenfundament. Ein großer Teil ökonomischer Aktivitäten, insbesondere die Dienstleistungen, gilt als schwer 
messbar, und selbst im Bereich der Herstellung der Informationstechnik bedarf es eines wissenschaftlich noch umstrittenen „hedonistischen“ Preisindexes, um die hohen Produktivitätssteigerungen statistisch ausweisen zu können. Die bisher offiziell ausgewiesenen Zuwächse liegen zudem noch unter denen der „goldenen“ 1960er Jahre.

Angesichts dieser Zweifel am Kern des New-Economy-Postulats erscheint die These von der spezifischen Globalisierungsform der US-Wirtschaft ebenso plausibel zu sein. Laut dieser These hält die Globalisierung in Form weitgehend liberalisierter Gütermärkte die Inflation unter Kontrolle. Die liberalisierten Finanzmärkte stellen ihrerseits die Mittel für Investitionen und für die inflationsdämpfenden Leistungsbilanzdefizite zur Verfügung. Im letzteren läge die Einzigartigkeit des US-Aufschwunges begründet, denn alle anderen Länder verfügen nicht über dieses hohe Vertrauen der internationalen Geldvermögensbesitzer in ihre Zahlungsfähigkeit. Allerdings ist dieses Vertrauen nicht grenzenlos. Eine Abkehr der internationalen Vermögensbesitzer vom US-Dollar und seine damit einhergehende Abwertung würde gemäß dieser These rasch zu harten zinspolitischen Maßnahmen führen.

Derzeit läßt sich noch nicht entscheiden, ob die „New Economy“ tatsächlich ein neues Akkumulationsregime darstellt oder ob es sich bloß um eine kurzfristige Sonderentwicklung handelt, die aus unterschiedlichen Gründen begrifflich überhöht und zu einer neuen Phase des Kapitalismus erklärt wurde. Als sicher erscheint mir nur, dass der US-amerikanische Boom der letzten Jahre ganz entscheidend von äußeren Bedingungen begünstigt wurde und somit in seiner Gänze nicht verallgemeinerbar ist. Künftige Forschung sollte nicht nur darauf abzielen, die Produktivitätsentwicklung besser statistisch zu erfassen, sondern auch die Effekte einer möglichen New Economy von den Effekten der spezifischen Globalisierungsformen der US-Wirtschaft sowohl analytisch als auch empirisch schärfer zu trennen. Gegenwärtig erfährt die New Economy angesichts des Rückgangs der Unternehmensgewinne sowie des hohen Leistungsbilanzdefizites der USA ihre erste harte Bewährungsprobe. Wahrscheinlich wird man schon bald genauer sehen können, ob es sich bei ihr tatsächlich um ein neues Akkumulationsregime handelt, das dann auch mit erheblichen Konsequenzen für die zukünftigen Auseinandersetzungen zwischen Kapital und Arbeit verbunden ist, oder ob dies nicht der Fall ist. 

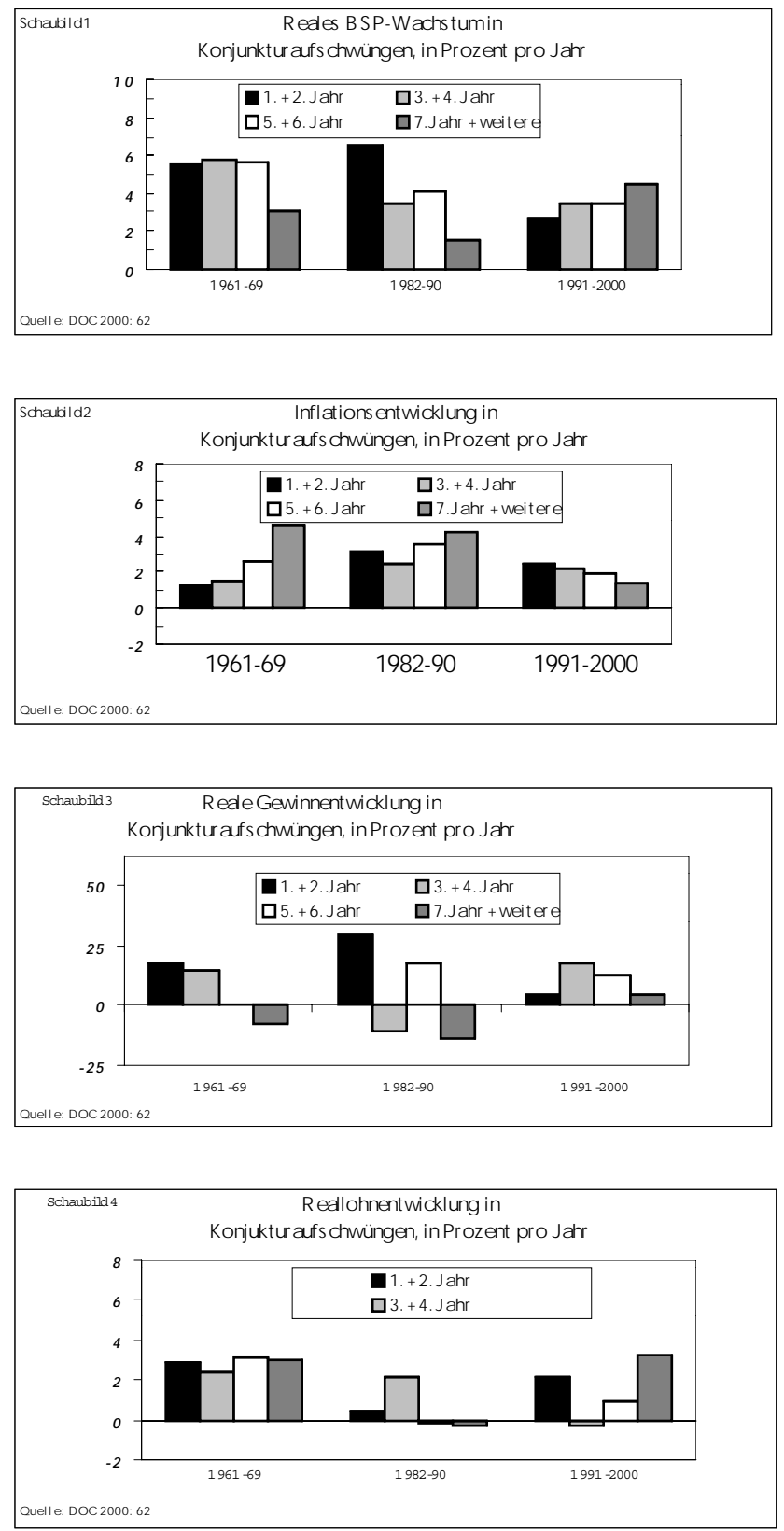
Schaubild 5

Produktivitätssteigerungen (pro Stunde) in

Konjunkturaufschwüngen pro Jahr,

Privatwirtschaft (ohne Landwirtschaft)

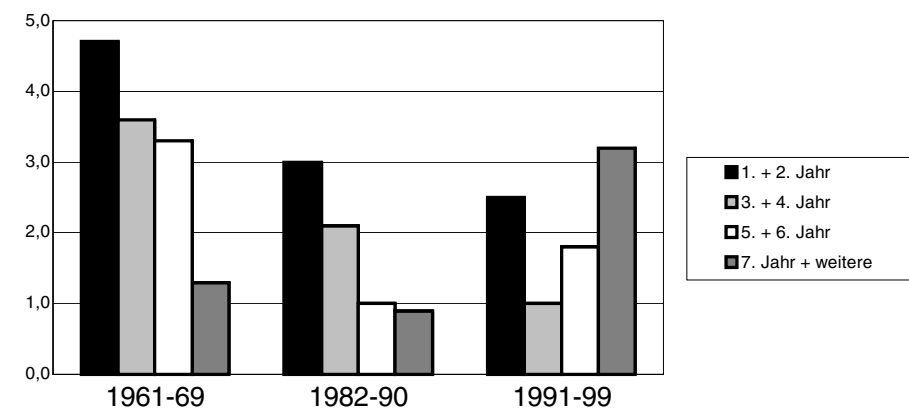

Quelle: DOC 2000: 33

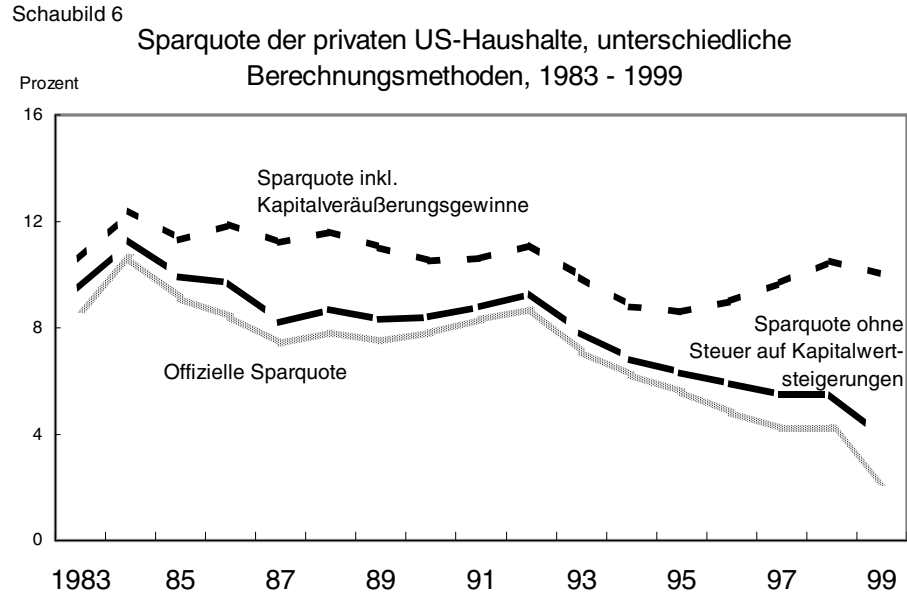

Quelle: Peach/ Steindel 2000: 4 


\section{Literatur}

Aglietta, Michel, 1979: A Theory of Capitalist Regulation. The US Experience, New York, NLB; Original: Régulation et crises du capitalisme, Paris (1976) Calmann-Lévy.

-, 2000: Ein neues Akkumulationsregime. Die Regulationstheorie auf dem Prüfstand, Hamburg, VSA.

Altvater, Elmar, Birgit Mahnkopf, 2000: „New Economy“ - nichts Neues unter dem Mond? in: WSI-Mitteilungen 53(12), 770-778.

Bassanini, Andrea, Stefano Scarpetta, Ignazio Visco, 2000: Knowledge, Technology and Economic Growth: Recent Evidence from OECD Countries, paper presented at the 150th Anniversary Conference of the National Bank of Belgium, „How to Promote Economic Growth in the Euro Area Brussels", 11 and 12 May.

Bergsten, Fred, 2000: Remarks in First Session of the Economic Summit, White House Press Release April 5, Washington, D.C.

Blinder, Alan S., Richard E. Quandt, 1997: Waiting for Godot: Information Technology and the Productivity Miracle?, Princeton University Department of Economics Working Paper, May.

BLS - Multifactor Productivity Trends, 1998: Press release Sept. 21, 2000. http://stats.bls.gov/mprhome.htm

Bluestone, Barry, 1999: Wall Street contra Main Street: Das US-amerikanische Wachstumsmodell, in: Lang, Mayer Scherrer (1999), 22-43.

BOC - Bureau of the Census, 2000: Report FT900 (CB-00-149), Foreign Trade Division, July, Exhibit 17.

Boyer, Robert, 1990: The Regulation School: A Critical Introduction, New York, Oxford University Press.

- , 2000: Is a Finance-led Growth Regime a Viable Alternative to Fordism? A preliminary Analysis, in: Economy and Society 29 (1) 111-145.

Brenner, Robert, 2000: The Boom and the Bubble, in: New Left Review 6, 5-43.

Brynjolfsson, Erik, and Lorin M. Hitt, 1998: Beyond the Productivity Paradox: Computers are the Catalyst for Bigger Changes, in: Communications of the ACM, August.

Castells, Manuel, 2000: The Rise of the Network Society (Information Age, Vol. I), 2nd edition, Oxford, UK, Blackwell.

Cole, R., Y.C. Chen, J.A. Barquin-Stolleman, E. Dulberger, N. Helvacian, J.H. Hodge, 1986: Quality-Adjusted Price Indexes for Computer Processors and Selected Peripheral Equipment, in: Survey of Current Business, 66(1), 41-50.

David, Paul A., 1999: Digital Technology and the Productivity Paradox, Ten Years Later What Have We Learned, and What Do We still Need to Know?, Stanford University \& All Souls College, Oxford papers presented at the Understanding the Digital Economy: Data, Tools, and Research, on May 25 \& 26, 1999 at the Department of Commerce in Washington, DC.

David, Paul, and G. Wright, 1999: Early Twentieth Century Growth Dynamics: An Inquiry into the Economic History of 'Our Ignorance', Stanford: SIEPR Discussion Paper No. 98-3.

Dean, Edwin R. 1999: The Accuracy of the BLS Productivity Measures, in: Monthly Labor Review, February, 24-34.

DOC 2000: U.S. Commerce Department, Economics and Statistics Administration, Office of Policy Development, Digital Economy 2000, Washington, D.C.

Eisner, Robert, 1998: The Economy is Booming. So Why Are Economists Glum?, in: The Wall Street Journal, July 29, editorial page.

FRB-Federal Reserve Board, 1999: Minutes of the Federal Open Market Committee June 29 30, 1999 (http://www.federalreserve.gov/FOMC/MINUTES/19990629.HTM).

Fuerbringer, Jonathan, 2001: Stocks Off for Year's First Week as Rate-Cut Rally Fades, in: New York Times, Jan. 6.

Gordon, Robert J., 1999a: Has the „New Economy” Rendered the Productivity Slowdown Obsolete? Northwestern University, Revised Version, June 14.

-, 1999b: Foundations of the Goldilocks Economy: Supply Shocks and the Time-Varying NAIRU, February 3, revision of the paper presented at Brookings Panel on Economic Activity, Washington, D.C., September 4, 1998.

- , 2000: Does the „New Economy“ Measure up to the Great Inventions of the Past? May 1, 
2000 draft of a paper for the Journal of Economic Perspectives.

Greenspan, Alan 1996: Testimony before the U.S. House of Representatives Committee on Banking and Financial Services, July 23.

- , 1998: Testimony before the Joint Economic Committee, U.S. Congress, June 10.

-, Alan, 2000: Remarks in Afternoon Session of White House Conference on the New Economy, White House Press Release April 5, Washington, D.C.

Griliches, Zvi. 1995. R\&D and Productivity: Econometric Results and Measurement Issues. In P. Stoneman, ed., Handbook of the Economics of Innovation and Technological Change. Oxford: Basil Blackwell.

Haltiwanger, John, Ron S. Jarmin, 2000: Measuring the Digital Economy, Conference Draft, Census Bureau, Washington, D.C.

Häußermann, Hartmut, 1999: Perspektiven der Dienstleistungsgesellschaft - Chancen für Frauen in: Lang, Mayer Scherrer (1999), 72-81.

Hay, Colin, 2001: Globalization, Economic Change and the Welfare State: the ,Vexatious Inquisition of Taxation '?, in: R. Sykes et al. (eds.), Globalization and the European Welfare States, Houndmills, Basingstoke: Palgrave Publ. (erscheint im Juni)

Herr, Hansjörg, Klaus Voy, 1989: Währungskonkurrenz und Deregulierung der Weltwirtschaft, Marburg, Metropolis.

Hübner, Kurt, 1989: Theorie der Regulation. Eine kritische Rekonstruktion eines neuen Ansatzes der Politischen Ökonomie, Berlin, edition sigma.

Huws, Ursula, 2000: Der Mythos der „Weightless Economy”, in: Das Argument 42(5/6) 646-660.

Jorgenson, Dale W., Kevin J. Stiroh, 2000: Raising the Speed Limit: U.S. Economic Growth in the Information Age, forthcoming in Brookings Papers in Economic Activity, May 1, p. 37 (http://www.economics.harvard.edu/ faculty/jorgenson/papers/dj_ks5.pdf).

Jürgens, Ulrich, Thomas Malsch, Knut Dohse, 1989: Moderne Zeiten in der Automobilfabrik, Berlin, Springer.

Lang, Sabine, Margit Mayer, Christoph Scherrer (Hg.), 1999: Jobwunder USA - Modell für Deutschland?, Münster, Westfälisches Dampfboot.

Lenz, Allen J., 2000: The US Current Account. A Sectoral Assessment of Performance and Prospects, October, Prepared for The US Trade Deficit Review Commission, Washington D.C.

Leonhardt, David, 2000: The Chaos at the Core of Prosperity, in: New York Times, November 5.

Lipietz, Alain, 1985: The Enchanted World. Inflation, Credit and the World Crisis, London, Verso Book.

Mishel, Lawrence, Jared Bernstein, John Schmitt, 2000: The State of Working America 2000-01, Economic Policy Institute, Ithaca, NY, Cornell University Press.

Moody, Kim, 1994: Pulled Apart and Pushed Together: Changes in the Workplace and the Transformation of the U.S. Working Class, in: Solidarity Discussion Bulletin, Preconvention series 1, April 1, 5-21.

-, 1997: Pulled apart and pushed together: Die US-Arbeiterklasse in der neuen Ökonomie Nordamerikas, in: B. Lüthje, C. Scherrer (Hrsg.), Zwischen Rassismus und Solidarität. Diskriminierung, Einwanderung und Gewerkschaften in den USA, Münster, Verlag Westfälisches Dampfboot.

Oliner, Stephen D., Daniel E. Sichel, 2000: The Resurgence of Growth in the Late 1990s: Is Information Technology the Story? Washington, DC: Federal Reserve Board, May.

Palley, Thomas, 1999: Arbeitslosigkeit und makroökonomische Weichenstellungen in: Lang, Mayer Scherrer (1999), 44-53.

Peach, Richard, Charles Steindel, 2000: A Nation of Spendthrifts? An Analysis of Trends in Personal and Gross Saving Current Issues in Economics and Finance, Federal Reserve Bank of New York, September, 6 (10).

Pitz, Karl H., 2000: The U.S. Trade Deficit: A View from Europe. An Evaluation for the Trade Deficit Review Commission of the U.S. Congress Part I: Analysis, October, Frankfurt and Washington D. C.

Piven, Frances Fox, 1999: Der marktfreundliche US-amerikanische Sozialstaat in: Lang, Mayer Scherrer (1999), 226-238.

Rich, Robert W., Donald Rissmiller, 2000: Understanding the Recent Behaviour of U.S. Inflation, in: Current Issues in Economics and Finance, Federal Reserve Bank of New York, July, 6 (8). 
Rohatyn, Felix, 1995: Cut and Be Prosperous, in: The Wall Street Journal, Nov. 30, A20.

Scherrer, Christoph, 1992: Im Bann des Fordismus. Die Auto- und Stahlindustrie der USA im internationalen Konkurrenzkampf, Berlin, Edition Sigma.

- , 1999: Globalisierung wider Willen? Die Durchsetzung liberaler Außenwirtschaftspolitik in den USA, Berlin, Edition Sigma.

Shiller, Robert J., 2000: Irrational Exuberance, Princeton, Princeton University Press.

Solow, Robert M., 1987: We'd Better Watch Out, in: New York Review of Books, July, 12, S. 36.

Uchitelle, Louis, March 12, 2000a: Productivity Finally Shows the Impact of Computers, New York Times, New York.

- , 2000b: Consumer Confidence Plunges, Especially Among the Affluent, in: New York Times, December 23.

Woodward, Bob, 2000: Maestro: Greenspan's FED and the American Boom, New York, Simon \& Schuster.

Zerdick, Axel, et al. 1999: Die Internet-Ökonomie: Strategien für die digitale Wirtschaft, Berlin, Springer. 